DOI: https://doi.org/10.24297/jam.v16i0.8113

\title{
Initial Value Problem for Stochastic Hyprid Hadamard Fractional Differential Equation
}

Mahmoud M. El-Borai

Department of Mathematics, Faculty of Science, Alexandria University,

Alexandria, Egypt

m_m_elborai@yahoo.com

Wagdy G. El-sayed

Department of Mathematics, Faculty of Science, Alexandria University, Alexandria, Egypt wagdygoma@Alexu.edu.eg

A. A. Badr

Department of Mathematics, Faculty of Science, Alexandria University, Alexandria, Egypt

badrzoo@yahoo.com

\section{A. Tarek S.A.}

Department of Mathematics, Faculty of Science, Alexandria University, Alexandria, Egypt ahmedsayed2890@gmail.com

\begin{abstract}
In this paper, we discuss the existence of solutions for a stochastic initial value problem of Hyprid fractional differential equations of Hadamardtype given by

$$
\begin{gathered}
{ }^{\mathcal{H}} D^{\alpha}\left(\frac{u(t)}{f(t, u(t))}\right)=b(t, u(t))+\sigma(t, u(t)) \dot{W}(t), \quad 1<t<T, \quad 0<\alpha \leq 1, \\
\left.{ }^{\mathcal{H}} \mathcal{J}^{1-\alpha} u(t)\right|_{t=1}=\xi,
\end{gathered}
$$

where ${ }^{\mathcal{H}} D^{\alpha}$ is the Hadamard fractional derivative, $f:[1, T] \times \mathcal{L}_{2}(\Omega) \rightarrow$ $\mathcal{L}_{2}(\Omega)$ and $b, \sigma:[1, T] \times \mathcal{L}_{2}(\Omega) \rightarrow \mathcal{L}_{2}(\Omega),{ }^{\mathcal{H}} \mathcal{J}^{(\cdot)}$ is the Hadamard fractional integral and $\xi$ be such that $\left(\mathbb{E}\left(\|\xi\|^{2}\right)\right)^{1 / 2}<\infty$, are investigated. The fractional calculus and stochastic analysis techniques are used to obtain the required results.
\end{abstract}

Keywords: Stochastic analysis, Hadamard-type fractional differential equations, Hyprid fractional differential equations, Stochastic differential equations. Mathematics subject classification: $60 \mathrm{H} 20,60 \mathrm{H} 15,60 \mathrm{H} 10,34 \mathrm{~A} 08,34 \mathrm{~B} 18$.

\section{Introduction}

In this paper, we study the existence of solutions for a stochastic initial value problem of Hyprid fractional differential equations of Hadamard-type given by

$$
{ }^{\mathcal{H}} D^{\alpha}\left(\frac{u(t)}{f(t, u(t))}\right)=b(t, u(t))+\sigma(t, u(t)) \dot{W}(t), \quad 1<t<T, \quad 0<\alpha \leq 1,
$$




$$
\left.\mathcal{H}^{1-\alpha} u(t)\right|_{t=1}=\xi,
$$

where ${ }^{\mathcal{H}} D^{\alpha}$ is the Hadamard fractional derivative, $f:[1, T] \times \mathcal{L}_{2}(\Omega) \rightarrow \mathcal{L}_{2}(\Omega)$ and $b, \sigma:[1, T] \times \mathcal{L}_{2}(\Omega) \rightarrow \mathcal{L}_{2}(\Omega),{ }^{\mathcal{H}} \mathcal{J}^{(\cdot)}$ is the Hadamard fractional integral.

In recent years, stochastic differential equations have become more and more important and interesting to researchers due to their successful and potential applications in various fields ([24], [25], [26], [27], [28], [29], [31], [32]), and the basic theories and results of stochastic differential equations can be found in [24].

By hybrid differential equation, we mean that the terms in the equation are perturbed either linearly or quadratically or through the combination of first and second types. Perturbation taking place in form of the sum or difference of terms in an equation is called linear. On the other hand, if the equation is perturbed through the product or quotient of the terms in it, then it is called quadratic perturbation. So the study of hybrid differential equation is more general and covers several dynamic systems as particular cases ([1]).

Fractional calculus and fractional-order differential equations have been applied in many fields of science and engineering, such as physics ([4]-[5]), chemical ([20]-[21]), etc. Actually, the concepts of fractional derivatives are not only generalization of the ordinary derivatives, but also it has been found that they can efficiently and properly describe the behavior of many physical systems (real-life phenomena) more accurately than integer order derivatives. Fractional differential equations involving Riemann-Liouville and Caputo-type fractional derivatives have extensively been studied by several researchers. However, the literature on Hadamard type fractional differential equations is not enriched yet. the fractional derivative due to Hadamard, introduce in 1892 [3], differs from the aforementioned derivatives in the sense that the kernel of the integral in the definition of the Hadamard derivative contains logarithmic function of arbitrary exponent. A detailed description of the Hadamard derivative and integral can be found in (e.g., [2]).

\section{Material and Methods}

This section contains some preliminary facts that we need in the sequel.

Let $(\Omega, \Im, \mathbb{P})$ be a probability space, where $\Omega$ is a sample space, $\Im$ is a $\sigma$ algebra and $\mathbb{P}$ is a probability measure. Let $\mathcal{C}=\mathcal{C}\left([1, T], \mathcal{L}_{2}(\Omega)\right)$ be the space of all second order stochastic processes which is mean square continuous on $[1, T]$, this space is a Banach space endowed with the norm $\|u\|_{\mathcal{C}}=\max _{t}\|u(t)\|_{2}$ where $\|u(t)\|_{2}=\left(\mathbb{E}\left(u^{2}(t)\right)\right)^{1 / 2}$.

Remark 1. For $y \in \mathcal{L}_{2}(\Omega)$, there holds the following Itô isometry property:

$$
\mathbb{E}\left\|\int_{1}^{t} y(s) d W(s)\right\|^{2}=\int_{1}^{t} \mathbb{E}\|y(s)\|^{2} d s .
$$

where $\{W(t)\}_{t \geq 0}$ is the Wiener (Brownian motion ) process

Definition 1. ([2]) The Hadamard fractional integral of order $\beta$ for a continuous function $f$ is defined as

$$
{ }^{\mathcal{H}} \mathcal{J}^{\beta} f(t)=\frac{1}{\Gamma(\beta)} \int_{1}^{t}\left(\ln \frac{t}{s}\right)^{\beta-1} f(s) d s, \quad \beta>0 .
$$


Definition 2. ([2]) The Hadamard derivative of fractional order $\beta$ for a continuous function $f$ is defined as

$$
{ }^{\mathcal{H}} D^{\beta} f(t)=\frac{1}{\Gamma(n-\beta)}\left(t \frac{d}{d t}\right)^{n} \int_{1}^{t}\left(\ln \frac{t}{s}\right)^{n-\beta-1} \frac{f(s)}{s} d s
$$

where $n-1<\beta<n, n=[\beta]+1$ and $[\beta]$ denotes the integer part of the real number $\beta$.

Theorem 1. ([2]) let $\alpha>0, n=-[-\alpha]$ and $0 \leq \nu<1$. Let $\mathcal{G}$ be an open set in $\mathcal{R}$ and let $h:(a, b] \times \mathcal{G} \rightarrow \mathcal{R}$ be a functions such that $h \in \mathcal{C}_{\nu, \ln }[a, b]$ for any $u \in \mathcal{G}$. then the problem

$$
\begin{gathered}
{ }^{\mathcal{H}} D^{\alpha} u(t)=h(t, u(t)), \quad \alpha>0, \\
{ }^{\mathcal{H}} \mathcal{J}^{\alpha-k} u\left(a^{+}\right)=b_{k} \in \mathcal{R}, \quad(k=1,2, \cdots, n \quad, n=-[-\alpha]) .
\end{gathered}
$$

satisfies the Volterra integral equation

$$
u(t)=\sum_{k=1}^{n} \frac{b_{k}}{\Gamma(\alpha-k+1)}\left(\ln \frac{t}{a}\right)^{\alpha-k}+\frac{1}{\Gamma(\alpha)} \int_{a}^{t}\left(\ln \frac{t}{s}\right)^{\alpha-1} h(s, u(s)) \frac{d s}{s} .
$$

In particular, when $0<\alpha \leq 1$, problem (3) is equivalent to the integral equation

$$
u(t)=\frac{b}{\Gamma(\alpha)}\left(\ln \frac{t}{a}\right)^{\alpha-1}+\frac{1}{\Gamma(\alpha)} \int_{a}^{t}\left(\ln \frac{t}{s}\right)^{\alpha-1} h(s, u(s)) \frac{d s}{s} .
$$

For more details (see e.g., [2]). From Theorem (1) we have the following lemma.

Lemma 1. ([13]) Given $u \in C\left([1, T], \mathcal{L}_{2}(\Omega)\right)$, the integral solution of the initial value problem

is given by

$$
\begin{gathered}
{ }^{\mathcal{H}} D^{\alpha}\left(\frac{u(t)}{f(t, u(t))}\right)=y(t), \quad 0<t<1, \\
\left.\mathcal{H}^{1-\alpha} u(t)\right|_{t=1}=\xi,
\end{gathered}
$$

$$
u(t)=f(t, u(t))\left(\frac{\xi}{\Gamma(\alpha)}(\ln t)^{\alpha-1}+\frac{1}{\Gamma(\alpha)} \int_{1}^{t}\left(\ln \frac{t}{s}\right)^{\alpha-1} y(s) \frac{d s}{s}\right), \quad t \in[1, T]
$$

the following lemma (fixed point theorem due to [1]) is the fundamental in the proof of our main result.

Lemma 2. ([1]) Let $S$ be a nonempty, closed convex and bounded subset of the Banach algebra $E$ and let $A: E \rightarrow E$ and $B: S \rightarrow E$ be two operators satisfying:

1. A is Lipschitzian with Lipschitz constant $k$,

2. B is completely continuous,

3. $x=A x B y \Rightarrow x \in S$ for all $y \in S$,

4. $M k<1$, where $M=\|B(S)\|=\sup \{\|B(x): x \in S\|\}$,

Then the operator equation $x=A x B x$ has a solution in $S$. 


\section{Main Result}

For $t \in[1, T]$, we define $u_{r}(t)=(\ln t)^{r} u(t), \quad r \geq 0$. Let $\mathcal{C}_{r}\left([1, T], \mathcal{L}_{2}(\Omega)\right)$ be the space of all continuous processes $u$ such that $u_{r} \in \mathcal{C}\left([1, T], \mathcal{L}_{2}(\Omega)\right)$ which is indeed a Banach space endowed with the norm $\|u\|_{\mathcal{C}}=\max _{t}\left\{(\ln t)^{r}\|u(t)\|_{2}\right\}$.

Theorem 2. Suppose that

(A1). The function $f$ is bounded continuous and there exists a positive bounded function $\varphi$ with bound $\|\varphi\|$ such that

$\mathbb{E}(\|f(t, x(t))-f(t, y(t))\|) \leq \varphi(t)\|x(t)-y(t)\|, \quad t \in[1, T], \quad x, y \in \mathcal{L}_{2}(\Omega) ;$

(A2). There exist a function $\psi \in \mathcal{C}\left([1, T], \mathcal{R}^{+}\right)$and continuous non-decreasing function $\zeta:[0, \infty) \rightarrow(0, \infty)$ such that for all measurable and continuous functions $b, \sigma$ the following conditions are satisfied

$$
\begin{aligned}
\mathbb{E}(\|b(t, x(t))\|) \leq \psi(t) \zeta(\|x\|), & t \in[1, T], \quad x \in \mathcal{L}_{2}(\Omega) ; \\
\mathbb{E}(\|\sigma(t, x(t))\|) \leq \psi(t) \zeta(\|x\|), & t \in[1, T], \quad x \in \mathcal{L}_{2}(\Omega) .
\end{aligned}
$$

(A3). There exists a number $\rho>0$ such that

$$
\rho \geq \mathcal{K}[\underbrace{\frac{\|\xi\|}{\Gamma(\alpha)}+\frac{\ln T}{\Gamma(\alpha+1)\|\psi\|} \zeta(\rho)+\frac{\mathcal{M}}{\Gamma(\alpha)}\|\psi\| \zeta(\rho)}] .
$$

where $\|f(t, x(t))\| \leq \mathcal{K}, \quad \forall(t, x) \in[1, T] \times \mathcal{L}_{2}(\Omega)$, $\mathcal{M}=T(\ln T)^{1-\alpha} \Gamma(2 \alpha-1) ;$

(A4). $\|\varphi\|[\underbrace{}]<1$.

Then the initial value problem (1) has at least one solution on $[1, T]$.

Proof. According to (Theorem 9.1, [1]) we split the proof into a sequence of steps.

Define a subset $S$ of $\mathcal{C}$ as

$$
S=\left\{u \in \mathcal{C}:\|u\|_{\mathcal{C}} \leq \rho\right\}
$$

where $\rho$ satisfies inequality (5).

Clearly, $S$ is closed, convex and bounded subset of the Banach space $\mathcal{C}$; by Lemma (2), the initial value problem (1) is equivalent to the integral equation

$$
\begin{aligned}
u(t) & =f(t, u(t))\left[\frac{\xi}{\Gamma(\alpha)}(\ln t)^{\alpha-1}+\frac{1}{\Gamma(\alpha)} \int_{1}^{t}\left(\ln \frac{t}{s}\right)^{\alpha-1} b(s, u(s)) \frac{d s}{s}\right. \\
& \left.+\frac{1}{\Gamma(\alpha)} \int_{1}^{t}\left(\ln \frac{t}{s}\right)^{\alpha-1} \sigma(s, u(s)) \frac{d W(s)}{s}\right]
\end{aligned}
$$

for $t \in[1, T]$. 
Define two operators $\mathcal{A}: \mathcal{C} \rightarrow \mathcal{C}$ by

$$
\mathcal{A} u(t)=f(t, u(t)), \quad t \in[1, T],
$$

and $\mathcal{B}: S \rightarrow \mathcal{C}$ by

$$
\begin{aligned}
\mathcal{B} u(t) & =\frac{\xi}{\Gamma(\alpha)}(\ln t)^{\alpha-1}+\frac{1}{\Gamma(\alpha)} \int_{1}^{t}\left(\ln \frac{t}{s}\right)^{\alpha-1} b(s, u(s)) \frac{d s}{s} \\
& +\frac{1}{\Gamma(\alpha)} \int_{1}^{t}\left(\ln \frac{t}{s}\right)^{\alpha-1} \sigma(s, u(s)) \frac{d W(s)}{s}, \quad t \in[1, T] .
\end{aligned}
$$

Then $u=\mathcal{A} u \mathcal{B} u$. We shall show that the operators $\mathcal{A}$ and $\mathcal{B}$ satisfy all the conditions of Lemma (2).

Step1. We first show that $\mathcal{A}$ is Lipschitz on $\mathcal{C}$. Let $u, v \in \mathcal{C}$. Then by (A1) we have

$$
\begin{aligned}
\left\|(\ln t)^{1-\alpha} \mathcal{A} u(t)-(\ln t)^{1-\alpha} \mathcal{A} v(t)\right\| & =(\ln t)^{1-\alpha}\|f(t, u(t))-f(t, v(t))\| \\
& \leq \varphi(t)(\ln t)^{1-\alpha}\|u(t)-v(t)\| \\
& \leq\|\varphi\|\|u-v\|_{\mathcal{C}} .
\end{aligned}
$$

for all $t \in[1, T]$. Taking the maximum over the interval $[1, T]$, we obtain

$$
\|\mathcal{A} u-\mathcal{A} v\|_{\mathcal{C}} \leq\|\varphi\|\|u-v\|
$$

for all $u, v \in \mathcal{C}$. So $\mathcal{A}$ is Lipschitz on $\mathcal{C}$ with Lipschitz constant $\|\varphi\|$.

Step2. The operator $\mathcal{B}$ is a completely continuous on $S$. We show that $\mathcal{B}$ is continuous on $S$. Then after some simple calculations we get

$$
\lim _{n \rightarrow \infty}(\ln t)^{1-\alpha} \mathcal{B} u_{n}(t)=(\ln t)^{1-\alpha} \mathcal{B} u(t) .
$$

for all $t \in[1, T]$. This shows that $\mathcal{B}$ is continuous on $S$. It sufficient to show that $\mathcal{B}(S)$ is a uniformly bounded and equi-continuous set in $\mathcal{C}$. First we have,

$$
\begin{aligned}
&(\ln t)^{1-\alpha}\|\mathcal{B} u(t)\|= \| \frac{\xi}{\Gamma(\alpha)}+(\ln t)^{1-\alpha} \frac{1}{\Gamma(\alpha)} \int_{1}^{t}\left(\ln \frac{t}{s}\right)^{\alpha-1} b(s, u(s)) \frac{d s}{s} \\
&+(\ln t)^{1-\alpha} \frac{1}{\Gamma(\alpha)} \int_{1}^{t}\left(\ln \frac{t}{s}\right)^{\alpha-1} \sigma(s, u(s)) \frac{d W(s)}{s} \| \\
& \leq \frac{\|\xi\|}{\Gamma(\alpha)}+\Delta_{1}+\Delta_{2}, \\
& \Delta_{1}=\frac{\|\psi\| \zeta(\rho)(\ln T)}{\Gamma(\alpha+1)} \\
& \Delta_{2}=\frac{\mathcal{M}\|\psi\| \zeta(\rho)}{\Gamma(\alpha)} .
\end{aligned}
$$

for all $t \in[1, T]$. Taking the maximum over the interval $[1, T]$, the above inequality becomes

$$
\|\mathcal{B} u\|_{\mathcal{C}} \leq \frac{\|\xi\|}{\Gamma(\alpha)}+\Delta_{1}+\Delta_{2}, \quad \forall u \in S .
$$


This show that $\mathcal{B}$ is uniformly bounded on $S$.

Next, we show that $\mathcal{B}$ is an equi-continuous in $\mathcal{C}$. Let $\tau_{1}, \tau_{2} \in[1, T]$ with $\tau_{1}<\tau_{2}$ and $u \in \mathcal{C}$. Then we have

$\left\|\left(\ln \tau_{2}\right)^{1-\alpha}(\mathcal{B} u)\left(\tau_{2}\right)-\left(\ln \tau_{1}\right)^{1-\alpha}(\mathcal{B} u)\left(\tau_{1}\right)\right\| \leq \frac{\|\psi\| \xi(\rho)}{\Gamma(\alpha)}\left\|\Xi_{1}+\Xi_{2}-\Xi_{3}-\Xi_{4}\right\|$

where

$$
\begin{aligned}
& \Xi_{1}=\int_{1}^{\tau_{2}}\left(\ln \tau_{2}\right)^{1-\alpha}\left(\ln \frac{\tau_{2}}{s}\right)^{\alpha-1} \frac{d s}{s} \\
& \Xi_{2}=\int_{1}^{\tau_{2}}\left(\ln \tau_{2}\right)^{1-\alpha}\left(\ln \frac{\tau_{2}}{s}\right)^{\alpha-1} \frac{d W(s)}{s} \\
& \Xi_{3}=\int_{1}^{\tau_{1}}\left(\ln \tau_{1}\right)^{1-\alpha}\left(\ln \frac{\tau_{1}}{s}\right)^{\alpha-1} \frac{d s}{s} \\
& \Xi_{4}=\int_{1}^{\tau_{1}}\left(\ln \tau_{1}\right)^{1-\alpha}\left(\ln \frac{\tau_{1}}{s}\right)^{\alpha-1} \frac{d W(s)}{s}
\end{aligned}
$$

And after some calculations, we will see that the right hand side of the above inequality tends to zero independently of $u \in S$ as $\tau_{2} \rightarrow \tau_{1}$. Therefore, it follows from Arzelá - Ascoli theorem that $\mathcal{B}$ is a completely continuous operator on $S$.

Step3. Let $u \in \mathcal{C}$ and $v \in S$ be arbitrary elements such that $u=\mathcal{A} u \mathcal{B} v$. Then we have

$$
\begin{aligned}
(\ln t)^{1-\alpha}\|u(t)\| & =(\ln t)^{1-\alpha}\|\mathcal{A} u(t)\|\|\mathcal{B} v(t)\| \\
& =\|f(t, u(t))\|\|\Upsilon\|
\end{aligned}
$$

where

$$
\begin{aligned}
\Upsilon & =\frac{\xi}{\Gamma(\alpha)}+(\ln t)^{1-\alpha} \frac{1}{\Gamma(\alpha)} \int_{1}^{t}\left(\ln \frac{t}{s}\right)^{\alpha-1} b(s, u(s)) \frac{d s}{s} \\
& +(\ln t)^{1-\alpha} \frac{1}{\Gamma(\alpha)} \int_{1}^{t}\left(\ln \frac{t}{s}\right)^{\alpha-1} \sigma(s, u(s)) \frac{d W(s)}{s}
\end{aligned}
$$

Therefore,

$$
(\ln t)^{1-\alpha}\|u(t)\| \leq \mathcal{K}\left[\frac{\|\xi\|}{\Gamma(\alpha)}+\Delta_{1}+\Delta_{2}\right]
$$

Taking the maximum over $[1, T]$, we obtain

$$
\|u\|_{\mathcal{C}} \leq \mathcal{K}\left[\frac{\|\xi\|}{\Gamma(\alpha)}+\Delta_{1}+\Delta_{2}\right] \leq \rho, \quad u \in S
$$

Step4. According to lemma (2), now we show that $M k<1$. This obvious by (A4), since we have

$$
\begin{aligned}
M & =\|B(S)\|=\sup \{\|B(x): x \in S\|\} \\
& \leq \frac{\|\xi\|}{\Gamma(\alpha)}+\Delta_{1}+\Delta_{2}, \quad k=\|\varphi\| .
\end{aligned}
$$

Thus all the conditions of Lemma (2) are satisfied and hence the operator equation $u=\mathcal{A} u \mathcal{B} u$ has a solution in $S$. In consequence, the problem (1) has a solution on $[1, T]$. 


\section{References}

[1] Bashir Ahmad, Ahmed Alsaedi, Sotiris K. Ntouyas, Jessada Tariboon, Hadamard-Type Fractional Differential Equations, Inclusions and Inequalities, Springer International Publishing, (2017).

[2] Anatoly A. Kilbas, Hari M. Srivastava, Juan J. Trujillo, Theory and Applications of Fractional Differential Equations, Elsevier, (2006).

[3] J. Hadamard, Essai sur l'etude des fonctions donnees par leur developpment de Taylor, J. Mat. Pure Appl. Ser, 8(1892), 101-186.

[4] Hilfer R, Application of fractional calculus in physics, New Jersey: World Scientific, (2001).

[5] Sabatier J, Agrawal O P, Machado J A T, Advances in fractional calculus, Dordrecht, The Netherlands: Springer, (2007).

[6] Omar K. Jaradat, Ahmad Al-Omari, Shaher Momani, Existence of the mild solution for fractional semi-linear initial value problems, Nonlinear Analysis, 69(2008), 3153-3159.

[7] J. Barrett, Differential equations of non-integer order, Canad.J.Math., 6(1954), 529-541.

[8] I. Podlubny, Fractional Differential Equations, Acadimic Press, New York, (1999).

[9] Mahmoud M. El-Borai, Some Probability densities and fundamental solutions of fractional evolution equations, Chaos Solitons and fractals, 14(2002), 433-440.

[10] M. M. El-Borai and R. M. Al-masroub, Exact Solutions for Some Nonlinear Fractional Parabolic Equations, no. 10, (2015), 106-122.

[11] M. M. El-Borai, K. E. S. El-Nadi, O. Labib, H. M. Ahmed, Semigroups and some fractional stochastic integral equations, Int. J. Pure Appl. Math. Sci., 3(1), (2006), 47-52.

[12] Hamdy M. Ahmed, Mahmoud M. El-Borai, Hilfer fractional stochastic integro-differential equations, Applied Mathematics and Computation, 331 (2018), 182-189

[13] Bashir Ahmed, Sotiris K. Ntouyas, Initial-Value Problems for Hyprid Hadamard Fractional Differential Equations, Electronic Journal of Differential Equations, no. 161, (2014), 1-8.

[14] Mainradi F., The fundamental solutions for the fractional diffusion-wave equation, Appl Math lett, 9(6)(1996).

[15] W. Feller, An Introduction to Probability Theory and Its Applications, Vol.II,John WileySons, New York, (1971).

[16] A. Friedman, Parial Differental Equations, Holt, Rinehart and Winston, New York, (1969). 
[17] I. M.Gelfand and G. E.Shilov, Generalized Functions, Vol.I: Properties and Operations, Nauka, Moscow, (1959).

[18] S. Kawatsu, Cauchy Problem for abstract evolution equations of Parabolic type, J. Math. Kyoto Univ.30(1990), no.1, 59-91.

[19] W. Wyss, The fractional diffusion equation, J. Math. Phys., 27(1986), no.11, 2782-2785.

[20] Khan N A, Ara A, Mahmood A., Approximate solution of time-fractional chemical engineering equations: a comparative study, Int. J. Chem. Reactor Eng., 8(2010) Article A19.

[21] Oldham K B., Fractional differential equations in electrochemistry, Adv. Eng. Softw., 41(1) (2010), 9-12.

[22] D. Delbosco, L. Rodino, Existence and uniqueness for a nonlinear fractional differential equation, J. Math. Anal. Appl. 204(1996), 609-625.

[23] J.H. He, Some applications of nonlinear fractional differential equations and their approximations, Bull. Sci. Technol. 15(2)(1999), 86-90.

[24] Oksendal B., Stochastic differential equations: an introduction with applications, Springer Science Business Media, (2013).

[25] Mao X., Stochastic differential equations and applications, Elsevier, (2007).

[26] El-Borai, M. M., On the correct formulation of the cauchy problem, Vestnik Moskov Univ. Scr. I Mat. Mch 23 (1968), 15-21.

[27] El-Borai, M.M., On some stochastic fractional integro-differential equations, Advances in Dynamical Systems and Applications, 1(2006), 49-57.

[28] El-Borai, M. M., El-Nadi, K.E-S., Ahmed, H.M., El-Owaidy, H.M., Ghanem, A.S., Sakthivel R., Existence and stability for fractional parabolic integro-partial differential equations with fractional brownian motion and nonlocal condition, Cogent Mathematics statistics, 1(2018), 146-166.

[29] El-Borai, M. M., El-said, K., A parabolic transform and some stochastic ill-posed problems, British Journal of Mathematics and Computer, (2015), 418-426.

[30] El-Nadi, K. E., On some stochastic parabolic differential equations in a Hilbert space, International Journal of Stochastic Analysis, 2(2005), 167-173.

[31] Mahmoud M. El-Borai and A. Tarek S.A., A unique Solution of Stochastic Partial Differential Equations with Non-Local Initial condition, Journal of Advances in Mathematics, 16(2019), 8226-8233.

[32] Mahmoud M. El-Borai and A. Tarek S.A., Existence and Uniqueness of Abstract Stochastic fractional-Order Differential Equations, Journal of Advances in Mathematics, 16(2019), 8280-8287. 